\title{
Dynamics of $\mathrm{OH} / \mathrm{IR}$ Stars in the Inner Galactic Bulge
}

\author{
ANDERS WINNBERG and MICHAEL LINDQVIST \\ Onsala Space Observatory, S-43992 Onsala, Sweden \\ and \\ HARM J. HABING \\ Sterrewacht Leiden, P.O. Box 9513, NL-2300 RA Leiden, The Netherlands
}

\begin{abstract}
Using the VLA at $1612 \mathrm{MHz}$ Lindqvist et al. (1992a) have found $134 \mathrm{OH} / \mathrm{IR}$ stars close to the Galactic Centre (GC). These stars plus 15 from Habing et al. (1983) have been used as probes of the gravitational potential to derive the mass distribution in the inner galactic bulge between $\approx 5$ to $\approx 100 \mathrm{pc}$ from the GC (Lindqvist et al., 1992b). In this paper we present a progress report of a dynamical model which we have applied to the data.
\end{abstract}

Key words: Galaxy (the): kinematics and dynamics of - Galaxy (the): center of - stars: longperiod variables - stars: OH/IR

\section{Introduction}

Estimates of the mass distribution within the inner regions of the Galaxy are crucial for our understanding of the dynamics in the inner few hundred pc and as a test of the existence of a massive central object. The gas dynamics in the inner region is complex and show non-circular motions (Genzel \& Townes, 1987). Studies of stellar kinematics within this region is essential since the mass distribution derived from the gas kinematics is uncertain and so is also the mass distribution estimated from the $2 \mu \mathrm{m}$ light distribution (Genzel \& Townes, 1987).

\section{Mass distribution}

We calculate the enclosed mass, $M(r)$, using Jeans' equations under the assumptions given in Lindqvist et al. (1992b). The resulting enclosed mass connects well with the values from McGinn et al. (1989) based on the $2.3 \mu \mathrm{m}$ CO bandhead for galactocentric distances of $\approx 0.3$ to $\approx 5 \mathrm{pc}$ (Fig. 1a). The latter work used the same method (Jeans' equations) under the same assumptions.

We have applied a model of the potential to our results and those of McGinn et al. (1989). We assume that the mass distribution is the sum of contributions from a point mass, $M_{\mathrm{c}}$, and a stellar cluster with a density dependence $\rho(r)=\rho_{0}$ for $r<r_{\mathrm{c}}$ (core radius), $\rho(r)=\rho_{0} r^{-\alpha_{1}}$ for $r_{\mathrm{c}}<r<r_{1}$, and $\rho(r)=\rho_{0} r^{-\alpha_{2}}$ for $r>r_{1}$. The "free parameters" are $M_{\mathrm{c}}, \rho_{0}, \alpha_{1}, \alpha_{2}, r_{\mathrm{c}}$, and $r_{1}$. Fig 1a shows an example of a reasonably good fit to the data. However, we emphasize that this is only one of several possible solutions. McGinn et al. (1989) did a similar analysis to their set of data. The circular velocity curve is shown in Fig. $1 \mathrm{~b}$.

We have created $l-b$ and $l-v$ diagrams by Monte Carlo calculations of stars, with certain orbital eccentricity and inclination distributions, injected into the potential. This is represented by gray-scale maps with the VLA sample stars superposed as comparison (Fig. 1c and 1d). We plan to proceed with similar modelling work. 

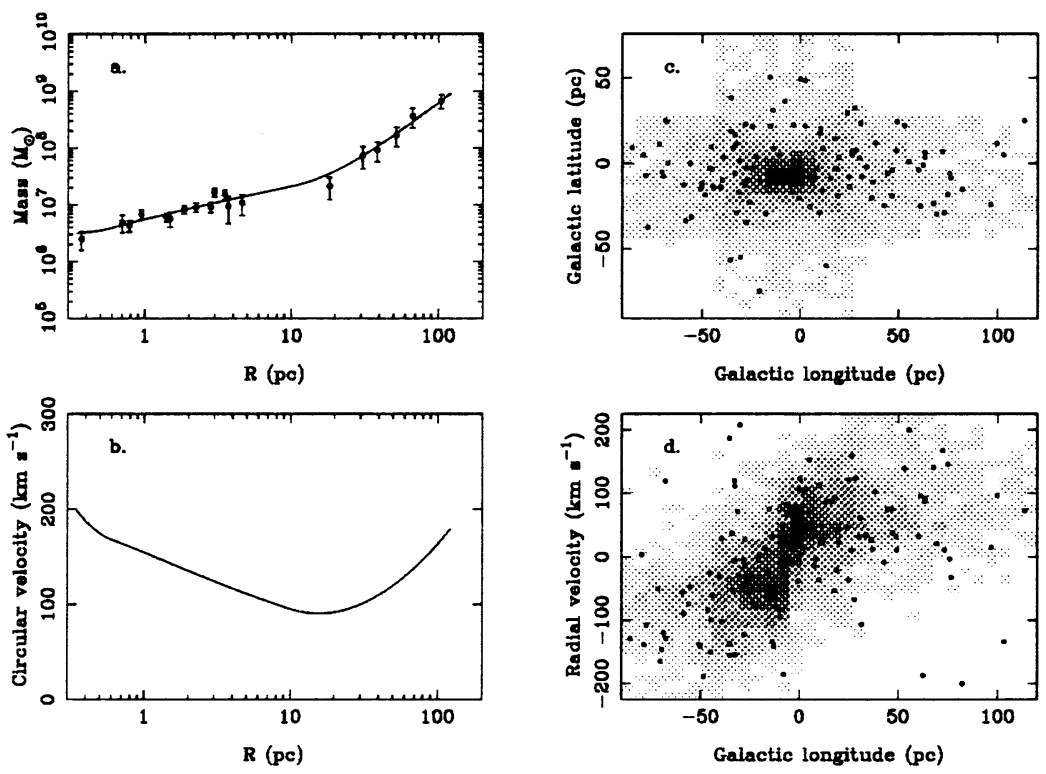

Fig. 1. An example of the model (a) given as a solid line with the following parameters $M_{\mathrm{c}}=3 \times 10^{6} \mathrm{M}_{\odot}, \rho_{0}=10^{6} \mathrm{M}_{\odot} \mathrm{pc}^{-3}, \alpha_{1}=-2.5, \alpha_{2}=-1.0, r_{\mathrm{c}} \approx 0.6 \mathrm{pc}, r_{1} \approx 10 \mathrm{pc}$. The circular velocity curve is shown in (b). The gray-scale plots represent distributions from Monte Carlo simulations ( $c$ and d). The VLA sample is denoted by dots.

It has become clear in the course of this survey that we have not got a statistically significant number of stars within a few parsecs $(\approx 5 \mathrm{pc})$ from the Galactic Centre. We therefore plan to carry out a more sensitive search in this area using the VLA. We also plan to extend the survey in galactic longitude and latitude.

In this work we have information on 3 (the position and the radial velocity) of the 6 variables of the problem. Thus the problem is heavily underdetermined. We therefore plan to search for $\mathrm{H}_{2} \mathrm{O}$ masers in these $\mathrm{OH} / \mathrm{IR}$ stars. Later we will try to determine their proper motions thus increasing the number of known variables to 5 .

\section{Acknowledgements}

This project is supported by the Swedish Natural Science Research Council (NFR).

\section{References}

Genzel R., Townes C.H., 1987, ARA\&A, 25, 377

Habing H.J., Olnon F.M., Winnberg A., Matthews H.E., Baud B., 1983, A\&A, 128, 230

Lindqvist M., Winnberg A., Habing H.J., Matthews H.E., 1992a, A\&AS, 92, 43

Lindqvist M., Habing H.J., Winnberg A., 1992b, A\&A, 259, 118

McGinn M.T., Sellgren K., Becklin E.E., Hall D.N.B., 1989, ApJ, 338, 824 